\title{
A Transmissão de Risco de Crédito em Cadeia de Suprimentos
}

Ricardo Reolon Jorge

Doutor e mestre em administração de empresas pela Fundação Getúlio Vargas de São Paulo - Departamento de Administração da Produção e de Operações - POI, MBA pela The University of Texas at Austin, engenheiro e técnico eletrônico. Professor da Universidade Federal do ABC - UFABC, São Paulo , Brasil

reolon.ricardo@ufabc.edu.br

Julio F. B. Facó

Graduado em Engenharia pelo Instituto Mauá de Tecnologia , pós-graduação em Marketing pela ESPM ,Mestrado em Administração de Empresas pela Fundação Getúlio Vargas - SP e Doutorado em Administração de Empresas com ênfase em Inovação em Cadeia de Suprimentos e Operações pela Fundação Getúlio Vargas SP .Professor da UFABC(Universidade Federal do ABC) vinculado aos Programas de Graduação em Engenharia de Gestão e Pós-Graduação em Engenharia e Gestão da Inovação, São Paulo , Brasil

julio.faco@ufabc.edu.br

Alexandre Andrade Acácio

Graduado em possui graduação em Eng.Elétrica - Ênfase em Energia e Automação (mestrado em Engenharia Elétrica (Automação) e doutorado (em Engenharia Elétrica (Automação) todos pela Escola Politécnica da Universidade de São Paulo. Professor em regime de dedicação exclusiva da UFABC .Docente do mestrado de Engenharia de Gestão da Inovação, São Paulo , Brasil

aacacio@ufabc.edu.br

Editor Científico: José Edson Lara

Organização Comitê Científico

Double Blind Review pelo SEER/OJS

Recebido em 26.12.2016

Aprovado em 08.06.2017

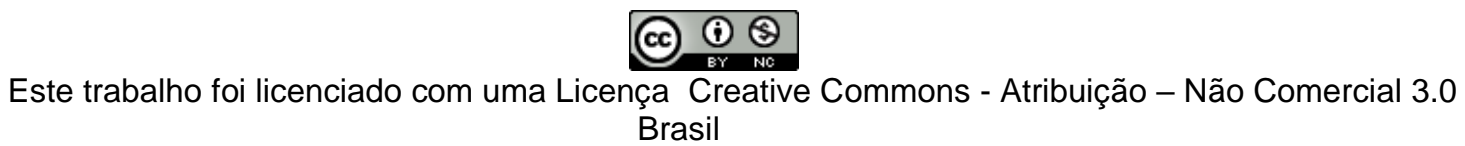




\section{Resumo}

O presente artigo tem por objetivo analisar a potencial transmissão de risco de crédito entre o comércio e a indústria na cadeia brasileira de calçados de couro. Para tal utilizaram-se as médias mensais da probabilidade de inadimplência de empresas do período de janeiro de 2008 a julho de 2013. Análises de séries temporais, testes de raiz unitária de Dickey Fuller Aumentado (ADF) e de Kwiatkowski, Phillips, Schmidt e Shin (KPSS), teste de cointegração de Johansen, modelo vetorial de correção de erros (VECM), causalidade de Granger, decomposição de erros da variância e função de resposta de impulso foram utilizados. Os resultados mostraram a existência de uma transmissão unidirecional do risco - da indústria para o comércio, bem como seu equilíbrio de longo prazo, confirmando a relevância da análise do risco de crédito das empresas em um contexto ampliado e que inclua os demais elos das cadeias de suprimentos.

Palavras-chave: Cadeia de suprimentos; risco de crédito; calçados de couro.

\section{Credit Risk Transmission in Supply Chains}

\section{Abstract}

This paper aims to analyze the potential credit risk transmission among commercial firms and industry of the Brazilian leather footwear supply chain. The data correspond to the monthly average of the delinquency index. The analyzed period embraces January 2008 to July 2013. Time series analysis, the Augmented Dickey Fuller (ADF) and the Kwiatkowski, Phillips, Schmidt e Shin (KPSS) unit root tests, the Johansen cointegration test, the Vector Error Correction Model (VECM), the Granger causality tests, the variance decomposition of the variance, and the estimation of impulse-response function were used. The results indicate the existence of a unidirectional transmission of credit risk at the supply chain studied, as well as its long-run equilibrium, confirming the importance of analyzing the credit risk of companies in an enlarged context that includes other firms in the supply chain.

Keywords: Supply chain; credit risk; leather footwear.

\section{La transmisión del riesgo de crédito en la cadena de suministro}

\section{Resumen}

Este artículo pretende analizar la potencial transmisión del riesgo de crédito entre el comercio y la industria en la cadena brasileña de zapatos de cuero. Para esto utiliza promedios mensuales de la probabilidad de impago de las empresas desde enero de 2008 a julio de 2013. Análisis de series de tiempo, raíz de la unidad prueba Augmented Dickey Fuller (ADF) y de Kwiatkowski, Phillips, Schmidt y Shin (KPSS), cointegración de Johansen prueba, modelo de corrección de error de vector (VECM) causalidad de Granger, descomposición de la varianza y el error de función de impulso respuesta se utilizaron. Los resultados mostraron la existencia de una transmisión unidireccional de riesgo comercio industria, así como su a largo plazo balance, confirmando la relevancia del análisis del riesgo de crédito de las empresas 
en un contexto ampliado y que incluye otros enlaces de las cadenas de abastecimiento.

Palabras clave: Cadena de suministro; riesgo de crédito; zapatos de cuero.

\section{Introdução}

O desempenho das organizações há muito está intimamente relacionado ao sucesso de suas cadeias de suprimentos. O Gerenciamento de Operações, com particular interesse na gestão de cadeias de suprimentos (Slack, Chambers, Johnston, \& Betts, 2013), abarca um complexo e amplo espectro de atividades e processos interconectados dentro e entre organizações distintas (Kanda \& Deshmukh, 2008).

Por outro lado, é interessante notar que, entre as diversas áreas de decisão fundamentadas na literatura especializada acerca de gestão de operações e cadeia logística, o risco, como tema de decisão, tem sido pouco explorado como já apontado por Wanke, Correa e Hijjar (2010).

Quando comparada à pesquisa em gerenciamento de operações, a pesquisa sobre risco de crédito de empresas e cadeias é um fenômeno mais recente (Barth, 2004). Os estudos mostram, em sua maioria, a ampla aplicação dos indicadores financeiros das empresas para a mensuração do risco de crédito, com pouca ou nenhuma aplicação de métricas quantitativas diretamente relacionadas às interfaces com clientes e fornecedores. Tal restrição impossibilita a compreensão e o entendimento do consequente impacto que decisões de empresas a montante ou a jusante em uma cadeia de suprimentos exercem sobre os resultados dos demais elos das cadeias em que estão inseridas.

Estabelecendo-se um paralelo entre estudos e técnicas de gerenciamento de operações aliadas à análise de risco de crédito, observa-se um hiato a ser preenchido nesta última que trate das interfaces das empresas em suas respectivas cadeias de suprimentos. A Tabela 1 ilustra esta questão.

Tabela 1

Primeiros estudos de gerenciamento de operações e risco de crédito

\begin{tabular}{l|l|l}
\hline & $\begin{array}{l}\text { Gerenciamento de } \\
\text { operações }\end{array}$ & \multicolumn{1}{c}{ Risco de crédito } \\
\hline Foco: Empresa & Final do século XIX. & Metade do século XX. \\
\hline Foco: Cadeia e Interfaces & $\begin{array}{l}\text { Metade do século } \\
\text { XX. }\end{array}$ & Final do século XX. \\
\hline
\end{tabular}

Fonte: Elaborada pelos autores 
Reconhecendo que questões associadas ao risco de crédito nas empresas ganham contornos novos quando analisadas sob a lente das teorias de cadeias de suprimentos (Bendoly, Donohue, \& Schultz, 2006), esta pesquisa tem como objetivo central identificar e explicar potenciais transmissões do risco de crédito entre o comércio e a indústria da cadeia de suprimentos brasileira de calçados de couro e busca responder às seguintes questões:

a) a transmissão de risco de crédito entre o comércio e a indústria é observável?

Se a transmissão de risco de crédito é observável:

b) em qual sentido se manifesta (a jusante, a montante ou bilateralmente)?

c) em qual horizonte de tempo se manifesta (curto e/ou longo prazos)?

Para tanto foram analisados os riscos de crédito de empresas da indústria brasileira de calçados de couro no período de janeiro de 2008 a junho de 2013.

$\mathrm{O}$ artigo foi dividido em quatro partes, além da Introdução. Na primeira realizou-se uma revisão sobre risco e, mais especificamente, sobre risco em cadeias de suprimentos. Na segunda, descrevem-se os procedimentos metodológicos. $\mathrm{Na}$ terceira, apresenta-se a análise dos dados levantados. Finalmente, são apresentadas as conclusões, limitações do presente estudo e sugestões para futuras pesquisas.

\section{Risco}

Usualmente o risco tem tido seu significado associado a eventos negativos que potencialmente reduzem o desempenho de processos e sistemas. Diversos são os pesquisadores que associam o significado de risco à chance ou possibilidade de perda (Vaughan, 1997; Bessis, 1998), às consequências de um evento ou saída específica (Ritchie \& Brindley, 2007) ou mesmo à mensuração da possibilidade de um resultado negativo (Emery \& Finnerty, 1997).

O risco tem basicamente duas dimensões no mundo dos negócios, segundo Ganguly (2013): probabilidade e severidade de um impacto negativo nos negócios. Dois tipos de risco podem ser classificados a partir destas dimensões. Um tem uma baixa probabilidade de ocorrência, é improvável que ocorra de uma forma similar e é difícil de se prever, mas, se ocorre, o impacto é muito significativo e dramático. Exemplos são desastres naturais, ataques terroristas e outros. O outro tem uma 
probabilidade relativamente alta de ocorrência, é repetitivo, previsível até um determinado ponto e tem um impacto menos dramático do que o primeiro. Exemplos são variações associadas à gestão da empresa e seu ambiente de negócios.

Os estudos acerca de riscos abordam uma grande variedade de áreas de estudo, sendo de nosso interesse especificamente sua aplicação em cadeias de suprimentos.

\subsection{Risco em cadeias de suprimentos}

O primeiro componente do risco em cadeias de suprimentos é a própria cadeia de suprimentos que pode ser definida como um sistema de organizações, pessoas, atividades, informações e recursos envolvidos na movimentação de um produto ou serviço do fornecedor ao cliente. Ela envolve as funções de compras, manufatura, estocagem, transporte, serviço ao cliente, planejamento da demanda e da oferta (Singh \& Wahid, 2014).

Para efeito deste estudo, considerar-se-á cadeia de suprimentos como o conjunto de duas ou mais organizações independentes e inter-relacionadas por meio de fluxos de bens, serviços, informações e recursos financeiros, objetivando a obtenção de resultados superiores para todos os elos envolvidos, tal como o fizeram Ghosh e Fedorowicz (2008). A função primária da cadeia de suprimentos é garantir a entrega pontual de bens e serviços com qualidade ao mercado (Stokes, 2008).

A dimensão de risco nas cadeias de suprimentos tem despertado a atenção de gestores e pesquisadores recentemente. Ganguly (2013) antecipa que a gestão de riscos em cadeias de suprimentos será uma das mais relevantes disciplinas e medida de competitividade nos próximos anos.

Hendricks e Singhal (2005) afirmam que os riscos em cadeias de suprimentos são rupturas nos fluxos naturais das cadeias e que se manifestam da forma mais variada, desde falhas na entrega de bens e serviços para elos a jusante nas cadeias, até restrições financeiras para o cumprimento de obrigações contraídas com fornecedores. Desta forma, a gestão adequada dos riscos em cadeias de suprimentos resulta em melhor desempenho financeiro e em vantagens competitivas para os agentes envolvidos, enquanto falhas nesta gestão podem ser muito custosas (Hauser, 2003). 
Contudo, para uma gestão adequada de riscos é necessário antes compreendê-los, tanto em termos de dimensões quanto de sua exposição às empresas em um contexto de cadeia de suprimentos. Enquanto alguns estudos apontam a existência de apenas dois tipos de riscos em cadeias de suprimentos aos quais as empresas estão expostas (Cousins, Lamming, \& Bowen, 2004) tecnológicos (excesso de confiança em um único ou limitado número de fontes de produtos, processos ou tecnologia) e estratégicos (excesso de confiança em um único ou limitado número de fornecedores) - outros pesquisadores vão além e classificam os riscos em 6 categorias (Olson \& Wu, 2010): naturais (desastres naturais, incêndios e epidemias); políticos (guerras, terrorismo, greves); concorrência e mercado (flutuações de preços, recessão econômica, flutuações nas taxas de câmbio, volatilidade da demanda, novas tecnologias); capacidade operacional (custos, seguros, desafios para aumento da capacidade, falência de fornecedores); operação interna (imprecisão nas previsões, acidentes com funcionários, efeito chicote, agilidade vs. flexibilidade); e riscos de sistemas de informação (falhas, inconsistência das informações, vírus/ bugs/ hackers).

Manuj e Mentzer (2008) focaram a discussão de riscos em torno de fornecimento, demanda, operacional e riscos de segurança. Kelly e Yang (2015) relacionam os riscos inerentes à cadeia de suprimentos e compras: riscos de contratos de custo fixo em que a organização compradora sabe o preço final com um elevado grau de certeza; risco de contratos de custos variáveis que envolvem os custos reais mais uma porcentagem de overhead e lucros; e risco de contratos de custos meta no qual os contratos de custo fixo e custos variáveis colocam o ônus da governança na organização compradora.

Não obstante tal tipificação, Faisal, Banwet e Shankar (2006) propõem algumas variáveis que potencialmente impactam o gerenciamento de risco de cadeias de suprimentos: compartilhamento de informações; agilidade e responsividade; confiança e colaboração entre parceiros na cadeia de suprimentos; segurança da informação; responsabilidade social; incentivos e políticas de divisão de receitas entre membros da cadeia; planejamento estratégico e análise de riscos. Outras pesquisas analisaram as fontes de incertezas que resultam em risco às empresas (Cucchiella \& Gastaldi, 2006). 
Autores como Christopher e Peck (2004), Manuj e Mentzer (2008), Bhattacharyya, Datta e Offodile (2010) também contribuíram com suas pesquisas acerca da compreensão do risco e avançaram propondo classificações e estratégias para mitigação de riscos em cadeias de suprimentos. Grötsch, Blome e Schleper (2013) propuseram uma abordagem gerencial proativa na redução e eliminação de riscos em cadeias de suprimentos.

Hyatt (2009) discutiu o dilema entre redução dos níveis de risco e cadeias de suprimentos enxutas apresentado no caso da empresa Cisco, enquanto Bonin e Cross (2010) e Mohanty e Dasgupta (2012) aprofundaram a discussão acerca de riscos em cadeias de suprimentos globais. A Tabela 2 apresenta uma síntese de estudos sobre risco em cadeias de suprimentos.

\section{Tabela 2}

Análise de estudos pregressos acerca do risco em cadeias de suprimentos.

Análise do Risco

Riscos como rupturas (falhas nas entregas).

Riscos tecnológicos, riscos de desenvolvimento de produtos e riscos estratégicos.

Riscos naturais, risco de concorrência e mercado, risco de capacidade operacional, riscos de operação interna e riscos de sistemas de informação.

Riscos de fornecimento, de demanda, operacional e de segurança.

Riscos macroeconômicos, riscos políticos, riscos competitivos e riscos de recursos.

Riscos em cadeias globais.

Riscos associados ao fluxo de material.

Risco associado ao fluxo de informação e de sistemas de informação.

Riscos associados ao fluxo de capital (recursos financeiros).

Riscos contratuais.

Variáveis que impactam o risco.

Tipos de incertezas associadas a riscos: internas e externas.

Fonte: elaborada pelos autores

Revista Gestão \& Tecnologia, Pedro Leopoldo, v. 17, n. 2, p. 134-159, mai./ago. 2017

\section{Autores}

Hendricks e Singhal (2005).

Cousins et al. (2004), Noël (2014)

Olson e Wu (2010).

Christopher e Peck (2004), Manuj e Mentzer (2008), Williamson (1979), Sheu et al (2006).

Ghoshal (1987).

Manuj e Mentzer (2008), Bhattacharyya et al. (2010), Christopher et al. (2011)

Zsidisin (2003), Lee et al. (1997), Huang e Gangopadhyay (2004).

Huang e Gangopadhyay (2004), Patnayakuni e Seth (2006), Childerhouse e Towill (2011), Ghosh e Fedorowicz (2008), Li (2002), Zsidisin (2003), Fawcett et al. (2007). Stonebraker e Liao (2004), Cucchiella e Gastaldi (2006), Bode et al. (2013), Schlegel e Trent (2012), Vicente (2003). Kelly e Yang (2015). Faisal et al. (2006). Cucchiella e Gastaldi (2006). 
Dentre os riscos apresentados, são de maior interesse para o presente estudo os riscos financeiros que são associados ao preço estável, hedging, cartas de crédito, pagamento de contas em dia, entre outros (Stonebraker \& Liao, 2004). Diversos pesquisadores também estudaram riscos financeiros em organizações e em cadeias de suprimentos (Cucchiella \& Gastaldi, 2006; Meulbrook, 2000; Schlegel \& Trent, 2012), bem como mecanismos para mitigá-los (Bode, Hübner, \& Wagner, 2013). Destaque para os grupos de riscos financeiros segundo a classificação adotada pelo Comitê de Supervisão Bancária da Basiléia: risco de mercado; risco legal; risco da reputação; risco operacional; risco de taxa de juros; risco de liquidez; risco de solvência; e, risco de crédito (Vicente, 2003).

\subsection{Riscos de crédito e probabilidade de inadimplência}

O risco de crédito está diretamente associado à probabilidade do não recebimento dos valores previamente contratados e decorrentes da entrega de um bem ou serviço. A relação credor e devedor é estabelecida a partir da promessa de pagamento do devedor em data futura ao credor. O possível não pagamento constitui o risco de crédito. Observa-se em Silva (2013): "[...] risco de crédito é o risco da contraparte deixar de cumprir suas obrigações relativas ao serviço da dívida; pode ser representado, também, pelo declínio do rating da contraparte, o que indica que a probabilidade de inadimplência aumentou [...]". A mesma abordagem é observada em Bessis (1998).

Os modelos de pontuação de crédito, também chamados de escoragem de crédito, medem objetivamente o risco de crédito, indicando percentualmente a probabilidade de inadimplência em um determinado horizonte de tempo, em geral 6 ou 12 meses. Para tal são aplicados modelos estatísticos para empresas pequenas e médias e também modelos julgamentais para corporações (Marins \& Neves, 2013). Nos modelos de escoragem de crédito são atribuídas pontuações às variáveis de decisão de crédito de um proponente, mediante a aplicação de técnicas estatísticas. Esses modelos visam, por meio de pesos (Caouette, Altman, \& Narayanan, 2009), a segregação de características (Lewis, 1994) que permitam distinguir adimplentes de inadimplentes, bons ou maus, desejáveis ou não, de acordo com a pontuação obtida por cada tomador de crédito. 
Por meio de estudos recentes acerca de risco de crédito e inadimplência (Marins \& Neves, 2013; Jacobson, Lindé, \& Roszbach, 2011; Fatemi \& Fooladi, 2006), propõe-se a probabilidade de inadimplência definida como a probabilidade de uma pessoa jurídica não cumprir suas obrigações creditícias após 90 dias do prazo acordado com o credor, e varia em uma escala de zero (nenhum risco da pessoa jurídica vir a inadimplir nos próximos 6 meses) a 100 pontos percentuais (empresa inadimplente), sendo que a condição de inadimplência já é caracterizada a partir de 70 pontos percentuais.

À luz do exposto, trataremos os conceitos probabilidade de inadimplência e risco de crédito como equivalentes, sendo o último adotado para as análises, discussões e conclusões do presente estudo.

\section{Procedimentos Metodológicos}

A base de dados utilizada no presente estudo foi gerada por um bureau de crédito líder no mercado nacional de informações creditícias. Contém os riscos de crédito de 112 mil empresas agrupadas em 40 setores econômicos, dentre os quais destaca-se o setor de calçados de couro com significativa relevância para a economia do país. Este setor é um dos maiores empregadores do país com $56 \%$ dos 328.521 trabalhadores formalmente registrados em 2013 no setor de calçados; $29 \%$ do total de calçados produzidos em $2011 ; 56,8 \%$ do valor total das exportações de calçados, ou US\$ 570 milhões; e, o maior valor médio por par entre os calçados atingindo a cifra de US\$30,12 em 2011 (Agência Brasileira de Promoção de Exportações e Investimentos [APEX], 2013).

A produção brasileira de calçados de couro está distribuída fundamentalmente entre os polos calçadistas do Rio Grande do Sul (Vale dos Sinos), interior de São Paulo (Jaú, Franca e Birigui), Nordeste (Paraíba, Ceará e Bahia), Santa Catarina (região de São João Batista) e Minas Gerais (Nova Serrana e Belo Horizonte) (Associação Brasileira das Indústrias de Calçados [ABICALÇADOS], 2009).

O setor apresentou expansão especialmente na década de 1990, exportando, somente para os Estados Unidos, cerca de 100 milhões de pares de sapatos por ano. No entanto, questões macroeconômicas e estruturais exerceram impacto negativo sobre os resultados do setor que viu suas exportações para os Estados Unidos reduzirem-se para cerca de 28 milhões de pares em 2009. Entre essas 
questões destacam-se a valorização cambial do real frente ao dólar, euro e yen, e a maior competição internacional, particularmente com a entrada da China como player internacional nesse setor.

Enquanto no período 2007 a 2013 as exportações retraíram 5,1\% em números de pares de sapatos e o preço médio do par em 17,5\%, as importações no mesmo período expandiram $4,6 \%$ e o preço médio do par $100 \%$.

Os calçados de couro, objeto do presente estudo, acompanharam a piora dos cenários do setor de calçados como um todo à medida que representaram cerca de $30 \%$ da produção brasileira de calçados em 2011. As exportações desse tipo de calçado despencaram de 115,9 milhões em 2001 para 18,5 milhões em 2012, levando o país do posto de 6 maior exportador em 2006 para 15e em 2011 (APEX, 2013).

A escolha da cadeia de suprimentos de calçados de couro justifica-se, assim, pela sua relevância para a economia nacional como grande geradora de empregos e de divisas, combinada à sensível interferência de cenários econômicos adversos que afeta sua capacidade de financiamento e adimplência. Para efeito do presente estudo, restringiu-se a análise da transmissão do risco de crédito aos elos do comércio e da indústria (Figura 1). Futuras pesquisas serão necessárias para ampliar a presente discussão aos demais elos a jusante e a montante.

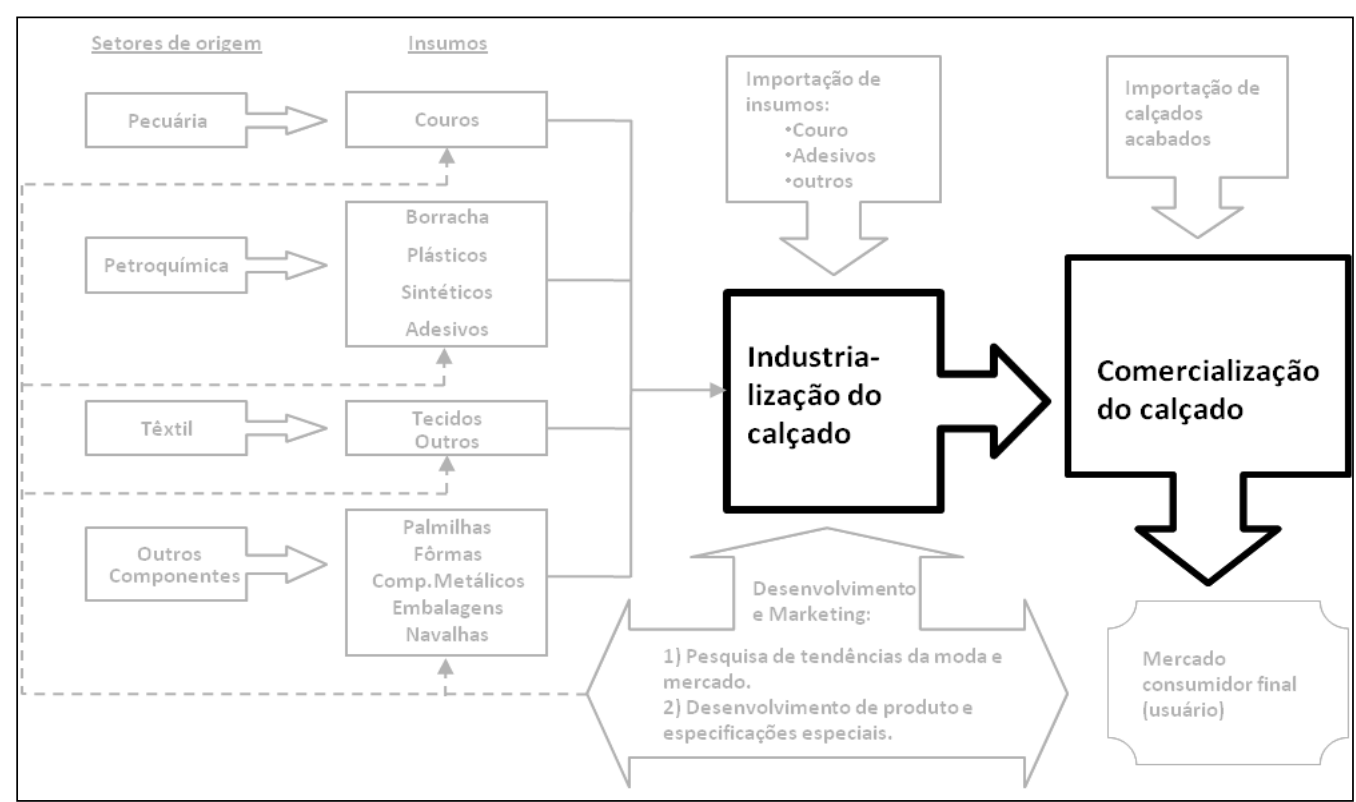

Figura 1

Elos selecionados da cadeia de suprimentos de calçados de couro Fonte: Elaborada pelos autores. 
O presente método inclui análises estatísticas de séries temporais e seguem a sequência: (1) análise da ordem de integração das variáveis, observando a estacionariedade das séries por meio dos testes ADF - Augmented Dickey-Fuller e KPSS - Kwiatkowski, Phillips, Schmidt e Shin; (2) relação causal entre as variáveis no curto prazo mediante o teste de causalidade de Granger; (3) cointegração entre as variáveis mediante o teste de cointegração de Johansen; (4) Modelo Vetorial de Correção de Erros (VECM); (5) função impulso-resposta pelo método de Cholesky; e (6) decomposição da variância dos erros de previsão.

O pacote estatístico/econométrico utilizado foi o EViews versão 8 SV.

\subsection{Seleção da Amostra e Cálculo das Variáveis}

Os dados básicos utilizados na pesquisa foram os riscos de crédito de empresas da cadeia de suprimentos brasileira de calçados de couro, no período de janeiro de 2008 a junho de 2013, ou 66 meses. Do total de 2.136 empresas amostradas, $86,94 \%$ são do comércio e $13,06 \%$ da indústria. A maior parte da amostra (1.470 observações) é formada por micro e pequenas empresas com faturamento máximo anual de $\mathrm{R} \$ 1,2$ milhões (Tabela 3).

\section{Tabela 3}

Faturamento Anual e Número de Empresas da Amostra

\begin{tabular}{|c|c|c|c|c|c|c|c|c|}
\hline \multirow{2}{*}{\multicolumn{2}{|c|}{$\begin{array}{l}\text { Faturamento anual } \\
\text { em } R \$ \text { milhões }\end{array}$}} & Mínimo & --- & $>1,2$ & $>4,0$ & $>25,0$ & $>50,0$ & \multirow{2}{*}{ Total } \\
\hline & & Máximo & 1,2 & 4,0 & 25,0 & 50,0 & 200,0 & \\
\hline \multirow{3}{*}{$\begin{array}{l}\text { Número } \\
\text { empresas }\end{array}$} & \multirow{3}{*}{ de } & Comércio & 1.291 & 485 & 73 & 8 & --- & 1.857 \\
\hline & & Indústria & 179 & 56 & 38 & 6 & --- & 279 \\
\hline & & Total & 1.470 & 541 & 111 & 14 & --- & 136 \\
\hline
\end{tabular}

Fonte: dados da pesquisa.

Os riscos de crédito das empresas de cada elo - indústria e comércio - foram transformados em logaritmo para que os valores dos próprios coeficientes obtidos fornecessem diretamente suas elasticidades, no presente estudo denominadas variáveis e representadas por lind e Icom respectivamente. As variáveis lind e Icom foram calculadas a partir da média mensal, em valores absolutos, dos riscos de crédito das empresas que formam a amostra e transformadas em logaritmo, totalizando 66 valores individuais para cada elo correspondentes ao período analisado (Tabela 4). 
Tabela 4

Modelo para Cálculo das Variáveis lind e Icom

\begin{tabular}{|c|c|c|c|c|c|}
\hline Mês & Empresa 1 & $\ldots$ & Empresa $\mathrm{n}\left({ }^{\mathrm{a}}\right)$ & $\begin{array}{l}\text { Média mensal } \\
\text { (valores absolutos) }\end{array}$ & $\begin{array}{c}\text { Média mensal } \\
(\log )\end{array}$ \\
\hline Jan/2008 & $\mathrm{RC}_{1,1}\left({ }^{\mathrm{b}}\right)$ & & $\mathrm{RC}_{\mathrm{n}, 1}$ & $\left(\mathrm{RC}_{1,1}+\ldots+\mathrm{RC}_{\mathrm{n}, 1}\right) / \mathrm{n}$ & lind $_{1}$ e lcom 1 \\
\hline Fev/2008 & $\mathrm{RC}_{1,2}$ & & $\mathrm{RC}_{\mathrm{n}, 2}$ & $\left(\mathrm{RC}_{1,2}+\ldots+\mathrm{RC}_{\mathrm{n}, 2}\right) / \mathrm{n}$ & $\operatorname{lind}_{2}$ e Icom $_{2}$ \\
\hline . & & & & & \\
\hline Jun/2013 & $\mathrm{RC}_{1,66}$ & & $\mathrm{RC}_{\mathrm{n}, 66}$ & $\left(\mathrm{RC}_{1,66}+\ldots+\mathrm{RC}_{\mathrm{n}, 66}\right) / \mathrm{n}$ & lind $_{66}$ e Icom $_{66}$ \\
\hline
\end{tabular}

Nota: $\left({ }^{\mathrm{a}}\right) \mathrm{n}$ : Indústria=279 empresas; comércio $=1.857$ empresas.

( $\left.{ }^{b}\right) \mathrm{RCi}$,j: Risco de Crédito da empresa i mês j.

Fonte: dados da pesquisa.

As curvas lind e Icom apresentam comportamentos semelhantes ao longo do período observado, sugerindo a potencial transferência de risco de crédito a ser confirmada pelas análises de séries temporais apresentados em Procedimentos Metodológicos (Figura 1).

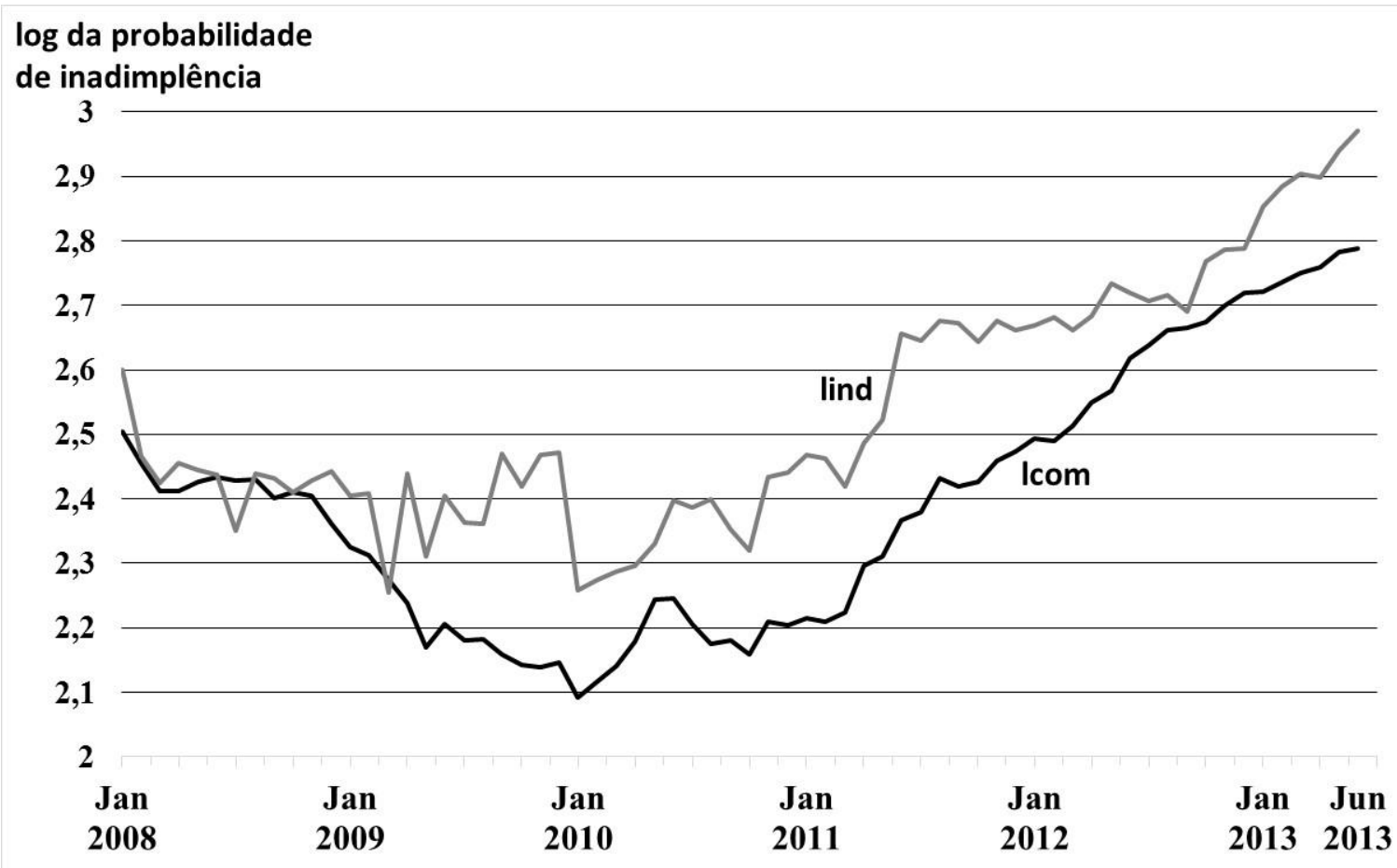

Figura 1

Risco de Crédito em Empresas (Indústria e Comércio).

Fonte: dados da pesquisa. 


\section{Resultados e Discussão}

A seguir são apresentados e interpretados os resultados das análises, obtidos com a aplicação dos procedimentos metodológicos propostos.

\subsection{Análise da ordem de integração das variáveis}

A estacionariedade é definida como a propriedade de um processo no qual os parâmetros estatísticos (média e desvio-padrão) do processo não se alteram com o tempo, e quando o valor da covariância entre dois períodos de tempo depende apenas da distância, do intervalo ou da defasagem entre dois períodos e não do próprio tempo em que a covariância é calculada. De forma contrária, os parâmetros de uma série temporal não estacionária ou processo estocástico variam com o tempo, apresentando possíveis tendências estocásticas em sua média ou variância. Muitas séries econômicas são não estacionárias, demandando alguma transformação para se tornarem estacionárias, tais como sua logaritmização e/ou aplicação do operador diferença, para eliminar a presença de tendência estocástica nas séries. Se a série temporal for diferenciada uma vez subtraindo-se $y_{t-1}$ de $y_{t}$ e torna-se estacionária $(\mathrm{I}(0))$, a série é então denominada como integrada de ordem 1 ou I(1) e possui, assim, uma raiz unitária.

O teste aplicado no presente estudo para verificar-se a ordem de integração das variáveis foi o teste de raiz unitária Augmented Dickey-Fuller (ADF) (Dickey e Fuller, 1979, 1981) com 2 defasagens segundo o critério de informação de Schwarz. Este teste tem como hipótese nula a presença de raiz unitária, ou seja, a não estacionariedade da série. O teste foi realizado em três modelos: com intercepto e com tendência, com intercepto, e sem intercepto e sem tendência.

Os resultados são apresentados na Tabela 5. Pode-se constatar que as variáveis em nível são não estacionárias, portanto, não se rejeita a hipótese nula de que há raiz unitária uma que vez que os valores calculados são menores em módulo do que seus respectivos valores críticos de $5 \%$ em todos os modelos analisados. 


\section{Tabela 5}

Resultado do Teste ADF em Séries Mensais de Probabilidade de Inadimplência em Nível

\begin{tabular}{llll}
\hline \multirow{2}{*}{ Séries } & \multicolumn{1}{c}{ Modelos } & \multicolumn{2}{c}{ Estatísticas do teste ADF } \\
\cline { 3 - 4 } & \multicolumn{2}{c}{$\mathbf{t}_{\text {cal }}$} & $\mathbf{T}_{(\mathrm{a}=\mathbf{0}, 05)}$ \\
\hline \multirow{2}{*}{ com } & Com intercepto e com tendência & $-1,701957$ & $-3,480463^{\mathrm{a}}$ \\
& Com intercepto & 1,020216 & $-2,906923^{\mathrm{a}}$ \\
& Sem intercepto e sem tendência & 1,183279 & $-1,946072^{\mathrm{a}}$ \\
\hline \multirow{2}{*}{ lind } & Com intercepto e com tendência & $-1,954043$ & $-3,481595^{\mathrm{a}}$ \\
& Com intercepto & 0,567817 & $-2,907660^{\mathrm{a}}$ \\
& Sem intercepto e sem tendência & 1,358792 & $-1,946072^{\mathrm{a}}$ \\
\hline
\end{tabular}

Fonte: dados da pesquisa.

Em contrapartida, os resultados apresentados na Tabela 6 mostram que as séries são estacionárias em primeira diferença ao nível de significância de $5 \%$ nos três modelos considerados.

\section{Tabela 6}

Resultados do Teste ADF para as Séries Mensais de Probabilidade de Inadimplência em Primeira Diferença

\begin{tabular}{llll}
\hline \multirow{2}{*}{ Séries } & \multicolumn{1}{c}{ Modelos } & \multicolumn{2}{c}{ Estatísticas do teste ADF } \\
\cline { 2 - 4 } dlcom & Com intercepto e com tendência & $\mathbf{t}_{\text {cal }}$ & $\mathbf{T}_{(\mathrm{a}=0,05)}$ \\
& Com intercepto & $-7,153741$ & $-3,481595^{\mathrm{a}}$ \\
& Sem intercepto e sem tendência & $-3,900493$ & $-2,908420^{\mathrm{a}}$ \\
\hline \multirow{2}{*}{ dlind } & Com intercepto e com tendência & $-3,733753$ & $-1,946072^{\mathrm{a}}$ \\
& Com intercepto & $-12,03712$ & $-3,481595^{\mathrm{a}}$ \\
& Sem intercepto e sem tendência & $-11,54617$ & $-2,907660^{\mathrm{a}}$ \\
\hline
\end{tabular}

Fonte: dados da pesquisa.

Estudos mostram que o teste de ADF é muito sensível à presença de valores atípicos e pela presença de parâmetros determinísticos (tendência e/ou constante), pelo número de observações na amostra e pelo número de defasagens utilizado nos testes, além da presença de quebras estruturais.

A existência de quebras estruturais pode dificultar a análise de uma série, pois é possível que o teste ADF mostre que uma série é não estacionária quando, na verdade, ela não o é. Assim, diz-se que este teste tem "baixo poder" contra processos estacionários. Um teste que diminui a incerteza decorrente do baixo poder do teste de raiz unitária ADF, é o teste de estacionariedade de Kwiatkowski, Phillips, Schmidt e Shin (1992), denominado KPSS. O teste KPSS é, segundo seus proponentes, uma forma de complementar a análise dos testes de raiz unitária 
tradicionais. Contrariamente aos testes usuais, tem como hipótese nula a estacionariedade da série e hipótese alternativa a não estacionariedade.

O teste foi realizado em dois modelos: com intercepto e com tendência, e com intercepto. Os resultados são apresentados na Tabela 7. Pode-se constatar que as variáveis em nível são não estacionárias, portanto, se rejeita a hipótese nula de que não há raiz unitária uma que vez que os valores calculados são maiores em módulo do que seus respectivos valores críticos de $5 \%$ em todos os modelos analisados.

\section{Tabela 7}

Resultados do Teste KPSS para as Séries Mensais de Probabilidade de Inadimplência em Nível

\begin{tabular}{|c|c|c|c|}
\hline \multirow{2}{*}{ Séries } & \multirow{2}{*}{ Modelos } & \multicolumn{2}{|c|}{ Estatísticas do teste KPSS } \\
\hline & & $t_{\mathrm{cal}}$ & $T_{(\alpha=0,05)}$ \\
\hline Icom & Com intercepto e com tendência & 0,259687 & $0,146000^{\mathrm{a}}$ \\
\hline & Com intercepto & 0,567071 & $0,463000^{a}$ \\
\hline lind & Com intercepto e com tendência & 0,248500 & $0,146000^{a}$ \\
\hline
\end{tabular}

Fonte: dados da pesquisa.

Em contrapartida, os resultados apresentados na Tabela 8 mostram que as séries são estacionárias em primeira diferença ao nível de significância de 5\% nos dois modelos considerados.

\section{Tabela 8}

Resultados do Teste KPSS para as Séries Mensais de Probabilidade de Inadimplência em Primeira Diferença.

\begin{tabular}{llll}
\hline \multirow{2}{*}{ Séries } & \multicolumn{1}{c}{ Modelos } & \multicolumn{2}{c}{ Estatísticas do teste KPSS } \\
\cline { 3 - 4 } & \multicolumn{1}{c}{$\mathbf{t}_{\mathrm{cal}}$} & $\mathbf{T}_{(\mathrm{a}=0,05)}$ \\
\hline dlcom & Com intercepto e com tendência & 0,114306 & $0,146000^{\mathrm{a}}$ \\
& Com intercepto & $0,854850^{\mathrm{b}}$ & $0,463000^{\mathrm{a}}$ \\
\hline \multirow{2}{*}{ dlind } & Com intercepto e com tendência & 0,073781 & $0,146000^{\mathrm{a}}$ \\
& Com intercepto & 0,459662 & $0,463000^{\mathrm{a}}$ \\
\hline
\end{tabular}

Fonte: $\left({ }^{\mathrm{a}}\right)$ dados da pesquisa

$\left({ }^{b}\right)$ o teste ADF (Tabela 6) aponta para a estacionariedade da série.

Com base nos resultados dos testes ADF e KPSS, conclui-se que as séries Icom e lind são estacionárias em primeira diferença I(0) e integradas em nível I(1).

\subsection{Relação causal entre as variáveis no curto prazo}

A existência de uma relação entre variáveis não significa que haja causalidade entre elas nem prova a direção de influência. Koop (2000) destaca que no caso de séries temporais: "[...] o tempo não corre para trás. Isto é, se A acontece antes de B, 
então é possível que $A$ cause $B$, contudo não é possível que B cause $A$ [...]”. Dessa maneira, os eventos do passado podem causar os eventos de hoje, enquanto os eventos futuros não podem.

A causalidade de Granger (1969) é um conceito específico sobre relações causais de curto prazo em séries temporais. A ideia é simples: a variável $X$ causa $Y$ se $Y$ pode ser melhor estimada utilizando valores passados de $X_{t}$ e $Y_{t}$ do que usando apenas valores históricos de $Y_{t}$. Para tal $Y_{t}$ deve ocorrer antes de $X_{t}+1$ e $Y_{t}$ conter informações relevantes para predizer $X_{t}+1$ que não são encontradas em um grupo com outras variáveis. $O$ fato de $Y$ Granger-causa $X$ não significa que há um mecanismo econômico em que valores futuros de $X$ são causados por $Y$, mas sim que $Y$ tem relevância para previsões de $X$.

Para a aplicação do teste de causalidade de Granger, determinou-se o número de defasagens a serem utilizadas. Para isso, empregou-se o Critério de Informação de Schwarz (SC), que resultou em um modelo auto regressivo para a segunda defasagem.

A distribuição $F$ (de Fisher) é aplicada para testar a hipótese nula $\left(\mathrm{H}_{0}\right)$ "X não causa, no sentido Granger, Y" e os resultados são apresentados na Tabela 9.

\section{Tabela 9}

Resultados do Teste de Causalidade de Granger em Primeira Diferença para as Séries Mensais de Probabilidade de Inadimplência

\begin{tabular}{cll}
\hline Hipótese Nula & \multicolumn{1}{c}{ Estatísticas do teste } \\
\cline { 2 - 3 } dlind não Granger-causa dlcom & F-statistic & Probabilidade \\
\hline dlcom não Granger-causa dlind & 4,58383 & 0,0142 \\
\hline
\end{tabular}

Fonte: dados da pesquisa.

A hipótese nula $\left(\mathrm{H}_{0}\right)$ de que a indústria não causa, no sentido Granger, o comércio, foi rejeitada, pois a probabilidade desta hipótese é 1,42\%, inferior ao valor crítico de $5 \%$. Em contrapartida, a hipótese nula $\left(\mathrm{H}_{0}\right)$ de que o comércio não causa, no sentido Granger, a indústria não foi rejeitada uma vez que a probabilidade desta hipótese ser verdadeira é 9,37\%, superior ao valor crítico de $5 \%$.

Conclui-se, assim, que há uma relação unidirecional de causalidade de curto prazo de indústria para comércio, ou seja, a probabilidade de inadimplência do 
comércio é influenciada, no curto prazo, pela probabilidade de inadimplência da indústria, mas não o contrário.

\subsection{Cointegração entre as variáveis}

A cointegração é um conceito que permite capturar a relação de equilíbrio entre as variáveis, ou seja, se duas variáveis caminham juntas no longo prazo, embora no curto prazo possam não apresentar o mesmo comportamento.

Dado que a análise da ordem de integração das variáveis mostrou que as duas variáveis, Icom e lind, são I(1), realizou-se o teste de cointegração de Johansen para verificar se essas variáveis tendem a uma situação de equilíbrio no longo prazo.

Para a aplicação do teste de cointegração, primeiramente determinou-se o número de defasagens a serem utilizadas a partir do critério de informação de Akaike (AIC) que apresentou menor valor para defasagem de ordem cinco.

A partir da observação gráfica das séries estudadas, observou-se a presença de uma tendência determinística que foi adotada para a realização dos testes do traço $\left(\lambda_{\text {trace }}\right)$ e do máximo autovalor $\left(\lambda_{\max }\right)$.

Os resultados indicam a existência de, pelo menos, um vetor de cointegração de acordo com ambos os testes, rejeitando a hipótese nula $\left(\mathrm{H}_{0}\right)$ ao nível de $5 \%$ de que não há vetor de cointegração. Os resultados podem ser visualizados na Tabela 10.

\section{Tabela 10}

Resultados do Teste do Traço de Máximo Autovalor de Johansen para as Séries Mensais de Probabilidade de Inadimplência

\begin{tabular}{lllllll}
\hline $\begin{array}{l}\text { Hipótese } \\
\text { Nula }\end{array}$ & $\begin{array}{l}\text { Eigen- } \\
\text { value }\end{array}$ & $\begin{array}{l}\text { Estatística } \\
\lambda_{\text {trace }} \text { calculado }\end{array}$ & $\begin{array}{l}\text { Estatística } \\
\lambda_{\text {trace }} \\
\text { crítico ao nalor } \\
\text { de 5\% }\end{array}$ & $\begin{array}{l}\text { Estatística } \lambda_{\text {max }} \\
\text { calculado }\end{array}$ & $\begin{array}{l}\text { Estatística } \lambda_{\text {max }} \\
\text { valor crítico ao } \\
\text { nível de 5\% }\end{array}$ \\
\hline $\mathrm{r}=0$ & 0,309022 & $22,50994^{\mathrm{a}}$ & 15,59471 & $22,17881^{\mathrm{a}}$ & 14,26460 \\
\hline $\mathrm{r}<=1$ & 0,005504 & 0,331129 & 3,841466 & 0,331129 & 3,841466 \\
\hline
\end{tabular}

Nota. $\left({ }^{a}\right)$ indica que a hipótese nula é rejeitada ao nível de significância de $5 \%$ Fonte: dados da pesquisa.

Tendo em vista que o sistema apresenta duas variáveis e um vetor de cointegração, o modelo a ser ajustado deve ser o Modelo Vetorial de Correção de Erros (VECM). Trata-se de um modelo de série temporal em primeiras diferenças que também contém um termo de correção de erro, que serve para introduzir duas séries I(1) no equilíbrio de longo prazo (Wooldridge, 2011). 


\subsection{Modelo Vetorial de Correção de Erros (VECM)}

O Modelo Vetorial de Correção de Erros (VECM) é um modelo dinâmico no qual o movimento das variáveis em quaisquer períodos está relacionado aos períodos anteriores no equilíbrio de longo- prazo. A cointegração provê os meios de separar as séries temporais em duas componentes - características do equilíbrio de longo-prazo e dinâmicas de desequilíbrio de curto-prazo. Assim, a especificação do Modelo de Correção de Erros é o meio mais eficiente de representar as propriedades de longo-prazo de um sistema, as propriedades de curto-prazo ou desequilíbrios e a natureza dos ajustamentos para o equilíbrio (Vavra \& Goodwin, 2005).

A equação de equilíbrio de longo-prazo é apresentada na Tabela 11. A equação indica que, mantendo tudo o mais constante, havendo um choque de $1 \%$ na probabilidade de inadimplência da indústria, haverá uma transmissão mais do que proporcional para o comércio de 1,32\%, caracterizando uma relação elástica no longo-prazo.

Tabela 11

Equação de Equilíbrio de Longo Prazo

\begin{tabular}{ccc}
\hline Modelo & $\begin{array}{c}\text { Número de } \\
\text { defasagens }\end{array}$ & $\begin{array}{c}\text { Equação de equilíbrio de longo prazo } \\
\text { Icom }_{t-1}=0,974174+1,32 \text { lind } t \\
(0,11385)[-11,6296]^{\mathrm{a}}\end{array}$ \\
\hline $\begin{array}{c}\text { Com intercepto e sem tendência } \\
\text { linear }\end{array}$ & 5 &
\end{tabular}

Nota. $\left({ }^{a}\right)$ (Erro-padrão); [Estatística $\left.t\right]$.

Fonte: dados da pesquisa

Desequilíbrios de longo prazo no comércio são corrigidos a uma taxa de $15,38 \%$ ao mês. Portanto, diante de desequilíbrios de curto prazo, a variável Icom demorará cerca de 6,5 meses para restabelecer seu respectivo desequilíbrio (Tabela 12).

Tabela 12

Taxa de Equilíbrio de Longo Prazo para o Comércio.

\begin{tabular}{lccc}
\hline \multicolumn{1}{c}{ Modelo } & $\begin{array}{l}\text { Número } \\
\text { defasagens }\end{array}$ & de & $\begin{array}{l}\text { Taxa mensal de estabilização do } \\
\text { Comércio após choque não antecipado }\end{array}$ \\
\hline $\begin{array}{l}\text { Com intercepto e sem tendência } \\
\text { linear }\end{array}$ & 5 & $-0,153845$ \\
\hline
\end{tabular}

Fonte: dados da pesquisa. 


\subsection{Função de Impulso-Resposta}

A Função de Impulso-Resposta mostra o nível de responsividade das variáveis endógenas no VECM quando um impulso é dado no termo de erro. Segundo esta função, um impulso dado sobre a variável $X$ não afeta somente a ela própria, mas também é transmitido para as demais variáveis.

A Figura 2 mostra que um choque não antecipado sobre a indústria causa uma volatilidade de significativa amplitude no comércio até o décimo mês, quando diminui lentamente até o décimo nono mês e finalmente se estabiliza. Portanto constata-se que um choque na indústria é absorvido ao longo do tempo pelo comércio, confirmando a estabilidade de longo-prazo do modelo.

Response to Cholesky One S.D. Innovations

Response of DLCOM to DLIND

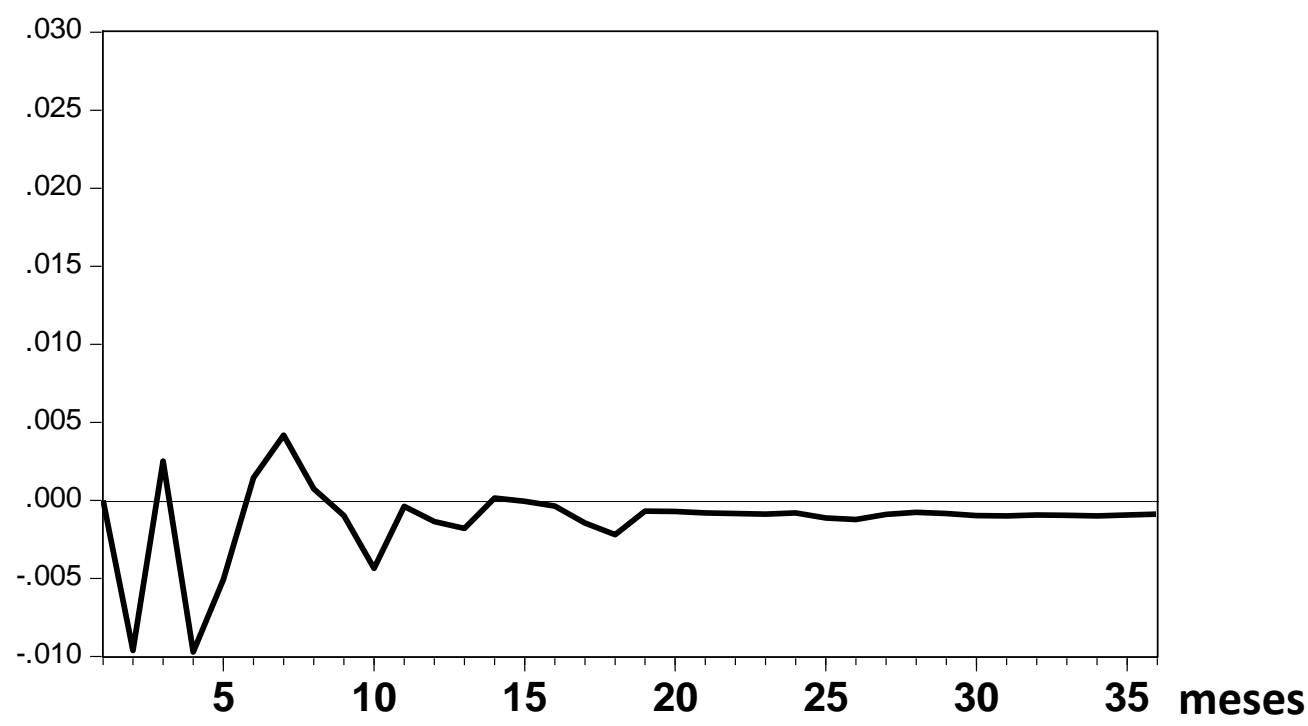

Figura 2

Função de Impulso-Resposta para as séries mensais de probabilidade de inadimplência, choque na Indústria e resposta do Comércio Fonte: dados da pesquisa.

\subsection{Decomposição da variância do erro}

Segundo Pinto (2007) "a decomposição de variância do erro de previsão é utilizada para descrever a dinâmica do sistema na abordagem VAR. Torna-se assim possível identificar a proporção da variação de uma variável devida a cada choque individual nas k variáveis do modelo". 
Os resultados referentes à decomposição da variância dos erros de previsão são apresentados na tabela 13, considerando um choque aplicado à indústria.

\section{Tabela 13}

Decomposição da Variância dos Erros de Previsão em Percentuais

\begin{tabular}{llll}
\hline \multirow{2}{*}{ Séries } & Período & Séries & \\
\cline { 3 - 4 } dlind & 1 & dlcom & dlind \\
& 12 & 10,63102 & 89,36898 \\
& 24 & 17,65043 & 82,34957 \\
& 36 & 21,95287 & 78,04713 \\
\hline
\end{tabular}

Fonte: dados da pesquisa.

Verifica-se que, após um choque não antecipado sobre a variável indústria, aproximadamente $90 \%$ da variância do seu erro de previsão decorre dela própria. Esta proporção reduz com o tempo, atingindo $74,12 \%$ após 36 meses.

Sob a ótica do comércio, observa-se que após 36 meses a indústria responde aproximadamente por $1 / 4$ da variância do seu erro de previsão, ou $25,89 \%$.

Conclui-se, assim, que um choque não antecipado na indústria tem um impacto bem definido sobre o comércio. Isto provavelmente acontece porque o setor industrial tem características de oligopólio quando comparado ao comércio, este bem mais pulverizado, em sua maioria com organizações de pequeno porte, e mais sensível às variações nos riscos de crédito dos demais atores da cadeia de suprimentos.

\section{Conclusões}

Os estudos acerca do risco em cadeias de suprimentos cresceram sobremaneira na última década, destacando-se os trabalhos de Schlegel e Trent (2012), Kelly e Yang (2015), Grötsch et al. (2013) e Christopher, Mena, Khan e Yurt (2011). A maioria dos estudos aprofundou a discussão nas dimensões tecnológicas, estratégicas, naturais, operacionais, econômicas e políticas dos riscos, porém pouca ênfase foi dada à questão da transmissão destes riscos entre os agentes das cadeias de suprimentos, em especial à transmissão dos riscos de crédito. $O$ presente estudo propõe uma discussão em torno desta questão, abordando a forma 
em que se dá a transmissão de risco entre os agentes comércio e indústria de uma mesma cadeia.

Primeiramente as variáveis risco de crédito do comércio e indústria foram convertidas em logaritmo para que os valores dos próprios coeficientes fornecessem diretamente as respectivas elasticidades. $O$ trabalho empírico em séries temporais pressupõe que a séria temporal subjacente seja estacionária (Gujarati, 2006, p. 635). Dessa maneira, aplicaram-se os testes ADF e KPSS e, com base nos seus resultados conclui-se que as séries contendo dados do elo comércio (Icom) e do elo indústria (lind) são estacionárias em primeira diferença $\mathrm{I}(0)$ e integradas em nível $\mathrm{I}(1)$, permitindo a realização das demais análises econométricas.

As relações de curto prazo foram verificadas por meio do teste de causalidade de Granger. Observou-se a existência de relações de curto prazo da indústria para o comércio. Em outras palavras, as variações de curto prazo da probabilidade de inadimplência na indústria afetam o comércio, porém estas mesmas relações não foram observadas no sentido contrário comércio - indústria.

Nas relações de longo prazo observou-se uma tendência de equilíbrio, tanto por meio do teste de cointegração de Johansen, do uso do VECM, quanto por meio da aplicação da função impulso-resposta pelo método de Cholesky. Os resultados mostraram que, havendo um choque de $1 \%$ na probabilidade de inadimplência da indústria, haverá uma transmissão mais do que proporcional para comércio de $1,32 \%$, caracterizando uma relação elástica no longo prazo e que desequilíbrios de longo prazo serão corrigidos a uma taxa de $15,38 \%$ ao mês, ou seja, a série comércio levará cerca de 6,5 meses para restabelecer seu respectivo equilíbrio.

Por sua vez, a análise da decomposição da variância dos erros de previsão mostrou que um choque não antecipado na indústria tem um impacto bem definido sobre o comércio, possivelmente porque o setor industrial de calçados de couro apresenta características de oligopólio quando comparado ao comércio. Este último, bem mais pulverizado e formado por micro e pequenas empresas, apresenta uma sensibilidade ao risco de crédito bem maior quando comparado à indústria.

Conclui-se, assim, que o presente estudo atingiu seus objetivos, demonstrando quantitativamente a existência da transmissão do risco de crédito entre os elos comércio e calçado da cadeia brasileira de calçados de couro, transmissão observável a jusante - da indústria para o comércio, tanto no curto como no longo prazo. 


\section{Limitações e Propostas para Futuras Pesquisas}

Sobre as limitações da presente pesquisa, há algumas questões que precisam ser esclarecidas. Primeiro, a escolha dos elos comércio e indústria, não considerando as dinâmicas dos demais elos a jusante e a montante na cadeia. Segundo, a escolha de uma única cadeia de suprimentos, calçados de couro, que tem dinâmicas específicas e diferentes de outras cadeias tais como a de vidros, têxtil e autopeças. E, finalmente, a não estratificação dos riscos de crédito por pequenas, médias e grandes empresas, considerando todos os valores para o cálculo do risco de crédito mensal, podendo assim haver vieses nos valores utilizados nas análises.

Para pesquisas futuras sugere-se a incorporação dos demais elos da cadeia de suprimentos, e a estratificação das análises segundo o porte das empresas (pequenas, médias e grandes). Outro aspecto interessante a ser pesquisado é a aplicação dos procedimentos metodológicos em outros tipos de cadeias de suprimentos e a comparação entre os resultados obtidos. Por último, por meio do agrupamento das cadeias de suprimentos em eficientes e responsivas, analisar a dinâmica da transmissão de risco de crédito segundo estes grupos e observar potenciais diferenças.

\section{Referências}

Agência Brasileira de Promoção de Exportações e Investimentos. (2013). Perfil Exportador do Setor Brasileiro de Calçados de Couro. Recuperado de http://www2.apexbrasil.com.br/media/estudo.

Associação Brasileira das Indústrias de Calçados. (2009). Resenha Estatística. Recuperado de http://www.abicalcados.com.br/documentos.

Barth, N. L. (2004). Inadimplência: construção de modelos de previsão. São Paulo: Nobel.

Bendoly, E., Donohue, K., \& Schultz, K. L. (2006). Behavior in operations management: assessing recent findings and revisiting old assumptions. Journal of Operations Management, 24, 737-752.

Bessis, J. (1998). Risk Management in Banking. London: John Wiley and Sons. 
Bhattacharyya, K., Datta, P., \& Offodile, O. F. (2010). The contribution of third-party indices in assessing global operational risks. Journal of Supply Chain Management, 46(4), 25-43.

Bode, C., Hübner, D., \& Wagner, S. M. (2010). Managing financially distressed suppliers: an exploratory study. Journal of Supply Chain Management, 50(4), 2443.

Boni, J., \& Cross, B. (2010). How to manage risk in global supply chain. Yvey Business Journal Reprints, 6.

Caouette, J., Altman, E., \& Narayanam, P. (2009). Gestão do risco de crédito: o próximo grande desafio financeiro. Rio de Janeiro: Qualitymark.

Childerhouse, P., Towill, D.R. (2011). Arcs of supply chain integration. International Journal of Production Research, 49(24), 7441-7468.

Christopher, M., Mena, C., Khan, O., \& Yurt, O. (2011). Approaches to managing global sourcing risk. Supply Chain Management: An International Journal, 16(2), 67-81.

Christopher, M., \& Peck, H. (2004). Building the resilient supply chain. International Journal of Logistics Management, 15(2), 1-13.

Cousins, P., Lamming, R. C., \& Bowen, F. (2004). The role of risk in environmentrelated initiatives. International Journal of Operations \& Production Management, 24(6), 554-65.

Cucchiella, F., \& Gastaldi, M. (2006). Risk management in supply chain: a real option approach. Journal of Manufacturing Technology Management, 17(6), 700-720.

Dickey, D., \& Fuller, W. A. (1979). Distribution of the estimates for autoregressive time series with a unit root. Journal of the American Statistical Association, 74(2), 427-431.

Dickey, D., \& Fuller, W. A. (1981). Likelihood ratio tests for autoregressive time series with a unit root. Econometrica, 49(5), 1507-1072.

Emery, D. R., \& Finnerty, J. D. (1997). Corporate Financial Management. New Jersey: Prentice Hall.

Faisal, M. N., Banwet, D. K., \& Shankar, R. (2006). Supply chain risk mitigation: modeling the enablers. Business Process Management Journal, 12(4), 535-552.

Fatemi, A., \& Fooladi, I. (2006). Credit risk management: a survey of practices. Managerial Finance, 32(3), 227-233.

Fawcett, S. E., Osterhaus, P., Magnan, G. M., Brau, J. C., \& McCarter, M. W. (2007). Information sharing and supply chain performance: the role of connectivity and willingness. Supply Chain Management: An International Journal, 12(5), 358-368. 
Ganguly, K. (2013). Book review: Supply Risk Assessment in SCM - Assessing Supply Side Risks. International Journal of Supply Chain Management, 2(4).

Ghosh, A., \& Fedorowicz, J. (2008). The role of trust in supply chain governance. Business Process Management Journal, 14(4), 453-470.

Ghoshal, S. (1987). Global strategy: an organizing framework. Strategic Management Journal, 8(5), 425-440.

Granger, C. W. J. (1969). Investigating causal relationship by econometric models and cross-spectral methods. Econometrica, 37(3), 424-439.

Grötsch, V. M., Blome, C., \& Schleper, M. C. (2013) Antecedents of proactive supply chain risk management - a contingency theory perspective. International Journal of Production Research, 51(10).

Gujarati, D. (2006). Econometria básica. Rio de Janeiro: Elsevier.

Hauser, L. M. (2003). Risk-adjusted supply chain management. Supply Chain Management Review, 7(6), 64-71.

Hendricks, K. B., \& Singhal, V. R. (2005). An empirical analysis of the effects of supply chain disruption on long-run stock price performance and equity risk of the firm. Production and Operations Management, 14(1), 35-52.

Huang, Z., \& Gangopadhyay, A. (2004). A Simulation Study of Supply Chain Management to Measure the Impact of Information Sharing Information. Resources Management Journal, 17(3), 20-32.

Hyatt, J. (2009). Ready for anything. CFO Magazine, 58-62.

Jacobson, T., Lindé, J., \& Rosbach, K. (2011). Firm default and aggregate fluctuations. International Finance Discussion Papers, Board of Governors of the Federal Reserve System, 1209.

Kanda, A. A., \& Deshmukh, T. S. (2008). Supply chain coordination: perspectives, empirical studies and research directions. International Journal of Production Economics, 115(2), 316-335.

Kelly, C., \& Yang, J. (2015). Improving the bottom line. Internal Auditor, 2, 54-59.

Koop, G. (2000). Analysis of economic data. Nova York: John Wiley and Sons. D.

Kwiatkowski, D., Phillips, P. C. B., Schmidt, P., \& Shin, Y. (1992). Testing the null hypothesis of stationarity against the alternative of a unit root: how sure are we that economic time series have a unit root? Journal of Econometrics, 54, 159178. 
Lee, H., Padmanabhan, V., \& Whang, S. (1997 Spring). The bullwhip effect in supply chains. Sloan Management Review.

Lewis, E. M. (1994). An introduction to credit scoring. Athena Press: United Kingdom.

Li, L. (2002). Information sharing in a supply chain with horizontal competition. Management Science, 48(9), 1196-1212.

Manuj, I., \& Mentzer, J. T. (in press). Global supply chain risk management. Journal of Business Logistics.

Marins, J. T. M., \& Neves, M. B. E. (2013) Inadimplência de crédito e ciclo econômico: um exame da relação no mercado brasileiro de crédito corporativo. Banco Central do Brasil.

Meulbrook, L. (2000). Total strategies for company-wide risk control. Financial Times, 9, 45-57.

Mohanty, R. P., \& Dasgupta, G.S. (2012). Identification of risk factors in globally outsourced software projects using logistic regression. International Journal of Supply Chain Management, 1(1).

Noël, P. (2014). The Apple watch: new tech, new risks. Risk Management Magazine, $12,10-11$.

Olson, D. L., \& Wu, D. D. (2010). A review of enterprise risk management in supply chain. Keybernets, 39(5), 694-706.

Patnayakuni, R., Rai, A., \& Seth, N. (2006). Relational antecedents of information flow integration for supply chain coordination. Journal of Management Information Systems, 23(1), 13-49.

Pinto, A. C. B., \& Vieira, F. V. (2008). Câmbio flexível e metas de inflação em países selecionados da América Latina: análise de vetores auto-regressivos (VAR). Revista Ensaios FEE, 29(1).

Ritchie, B., \& Brindley, C. (2007). Supply chain risk management and performance: a guiding framework for future development. International Journal of Operations and Production Management, 27(3), 303-322.

Schlegel, G. L., \& Trent, R. J. (2012). Risk management: welcome to the normal. Supply Chain Management Review, 16(1), 12-21.

Sheu, C., Yen, H. R., \& Chae, B. (2006). Determinants of supplier-retailer collaboration: evidence from an international study. International Journal of Operations and Production Management, 26(1), 24-49.

Silva, J. P. (2013). Gestão e análise de risco de crédito. São Paulo: Atlas. 
Singh, G., \& Wahid, N. A. (2014). Supply chain risk management: a review. International Journal of Supply Chain Management, 3(3).

Slack, N., Chambers, S., Johnston, R., \& Betts, A. (2013). Gerenciamento de operações e de processos: princípios e práticas de impacto estratégico. São Paulo: Bookman.

Stokes, R. (2008). Understanding supply chain. Risk Management, 54-57.

Stonebraker, P. W., \& Liao, J. (2004). Environmental turbulence, strategic orientation: modeling supply chain integration. International Journal of Operations \& Production Management, 24.

Vaughan, E. J. (1997). Risk Management. New Baskerville: John Wiley and Sons.

Vavra, P., \& Goodwin, B. K. (2005). Analysis of price transmission along the food chain, OECD Food, Agriculture and Fisheries Working Papers, 3.

Vicente, E. F. R. (2003). Modelo para avaliação de risco de crédito de municípios brasileiros. (Tese de Doutorado em Administração de Empresas, Faculdade de Economia, Administração e Contabilidade da Universidade de São Paulo, São Paulo, Brasil).

Wanke, F. P., Correa, H.,\& Hijjar, M. (2010). Establishing the relationship between logistics complexity and supply chain objectives and decision areas in large companies operating in Brazil. Journal of Operations and Supply Chain Management, 3, 34-54.

Williamson, O. E. (1979). Transaction Cost Economics: the governance of contractual relations. New York: The Free Press.

Wooldridge, J.M. (2011). Introdução à econometria: uma abordagem moderna. São Paulo: Cengage.

Zsidisin, G. A. (2003). Managerial perceptions of supply risk. The Journal of Supply Chain Management, 39(4), 14-26. 